\title{
Impact of Physical Inactivity on the Risk of Disability and Hospitalization in Older Patients with Advanced Lung Cancer
}

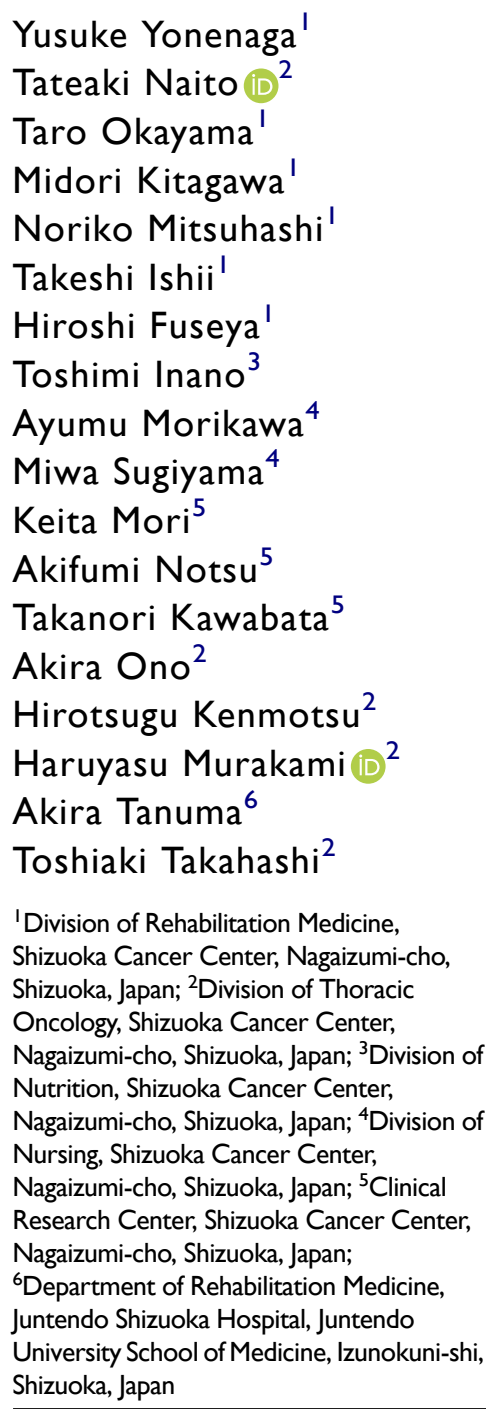

Correspondence: Tateaki Naito Division of Thoracic Oncology, Shizuoka Cancer Center, 1007, Shimonagakubo, Nagaizumi-cho, Shizuoka, 4 I I-8777, Japan Tel +8I-55-989-5222

Fax +8I-55-989-5783

Email t.naito@scchr.jp
Purpose: This prospective observational study aimed to explore the influence of physical inactivity during initial chemotherapy on the risk of disability and hospitalization in later life among older patients with advanced non-small-cell lung cancer (NSCLC).

Patients and Methods: Patients aged 70 or above who were scheduled to receive first-line chemotherapy for newly diagnosed advanced NSCLC were recruited for the study. An electronic pedometer was used to measure daily steps; based on the change rate (cutoff: $-12.5 \%$ ) from pretreatment to $12 \pm 4$ weeks after enrolment, patients were classified as active or inactive. The Barthel Index estimated activities of daily living. We compared disabilityfree survival time, mean cumulative functions of hospital stays, and medical costs, between the active and inactive groups.

Results: Among the 29 patients enrolled, 21 were evaluable. Compared with active patients (n $=11)$, inactive patients $(\mathrm{n}=10)$ showed shorter disability-free survival (6.4 vs 19.9 months, $p<$ 0.05 ) and tended to have longer hospital stays (23.7 vs 6.3 days/person) and higher inpatient care cost ( $¥ 1.6$ vs $¥ 0.3$ million/person [US\$16,000 vs US\$3000/person]) during the first year. Conclusion: Physical inactivity during initial chemotherapy may be a risk factor for developing disability and requiring hospitalization in later life for older patients with advanced NSCLC. Our findings may indicate the need for lifestyle interventions with multidisciplinary teams, which include physicians, nurses, and physiotherapists, for older patients with advanced lung cancer during an active cancer treatment. A large-sample-sized study is needed to validate our findings.

Keywords: non-small-cell lung cancer, older patients, physical activity, disability-free survival, medical cost, length of hospital stay

\section{Introduction}

The number of older patients with advanced lung cancer has been increasing because of global population aging ${ }^{1}$ and a high proportion of metastatic diseases at the time of diagnosis of lung cancer. ${ }^{2}$ Further, patients with advanced cancer now live longer because of advances in anticancer treatments, such as molecular targeted therapies and immunotherapies. ${ }^{3}$ Thus, these medical conditions may become an economic burden on society. In Japan, approximately half (57\%, ¥3.2 hundred million [US\$3.2 million]) of the annual national medical costs for tracheal, bronchial, and lung cancers were associated with older individuals aged 70 years or older, and the majority of the costs $(64 \%, ¥ 2.0$ hundred million [US\$2.0 million]) were associated with their inpatient care. ${ }^{4}$ 
Older patients are predisposed to sarcopenia ${ }^{5}$ and decreased physical function, ${ }^{6}$ which are highly associated with disability. ${ }^{7}$ Additionally, older patients with advanced lung cancer are prone to multiple comorbidities, ${ }^{8}$ malnutrition, ${ }^{9}$ muscle depletion, ${ }^{10}$ and cancer cachexia, ${ }^{11,12}$ thereby resulting in progressive physical inactivity. ${ }^{13,14}$ These negative characteristics account for this population's vulnerability and increase their medical dependency from an early cancer trajectory. ${ }^{12}$ This complexity in pathogenesis indicates a need for multidisciplinary approaches to prevent worsening physical conditions during cancer treatment in this population. ${ }^{15}$ However, there are few previous reports on the longitudinal changes in physical activity in patients with advanced lung cancer. ${ }^{14}$ Furthermore information on when physical inactivity occurs and how it affects functional and socioeconomic outcomes is limited. Accordingly, we conducted this prospective observational study to explore the influence of physical inactivity during initial chemotherapy on the risk of disability and hospitalization in later life among older patients with advanced non-small-cell lung cancer (NSCLC).

\section{Materials and Methods}

\section{Patient Selection}

This prospective longitudinal observational study was conducted from February 2014 to October 2017 at the Shizuoka Cancer Center, Japan. To be eligible, patients had to: (1) have histologically or cytologically proven metastatic or postoperative recurrent NSCLC; (2) be aged 70 years or older; (3) be scheduled to receive firstline systemic chemotherapy; (4) not have undergone previous systemic chemotherapy or thoracic radiotherapy (adjuvant chemotherapy was not counted as prior chemotherapy); (5) have an Eastern Cooperative Oncology Group performance status (PS) of $0-1$; (6) be able to ambulate, read, and respond to questions without assistance; and (7) have an expected survival of more than twelve weeks. Patients with severe psychiatric disorders, active infectious diseases, unstable cardiac disease, or untreated symptomatic brain or bone metastases hindering accurate assessment were excluded. All patients provided their written informed consent. The study was approved by the Shizuoka Cancer Center's institutional review board (Shizuoka, Japan) and registered on the University Hospital Medical Information Network Clinical Trials Registry in Japan (trial registration number:
UMIN000012845). All assessments were carried out under the ethical principles outlined in the Declaration of Helsinki.

\section{Patient Enrolment and Data Collection}

The study period was defined as the duration from the enrolment date to either the final visit or the cutoff date (August 31, 2018). Daily steps (steps/day) and duration of moderate to vigorous physical activity (MVPA, in minutes) were assessed at pretreatment and $12 \pm 4$ weeks (T2) after enrolment. Body mass index $\left(\mathrm{kg} / \mathrm{m}^{2}\right)$, lumbar skeletal muscle index $\left(\mathrm{cm}^{2} / \mathrm{m}^{2}\right)$, incremental shuttle walking distance $(\mathrm{m})$, and hand-grip strength $(\mathrm{kg})$ were assessed at pretreatment. Pretreatment assessments were performed during the period from enrolment to the first chemotherapy session. The attending physicians and physiotherapists performed all assessments. The best response to chemotherapy was evaluated by the Response Evaluation Criteria in Solid Tumors version 1.1. ${ }^{16}$

\section{Assessment of Physical Activity}

Daily steps and intensity of physical activity were continuously measured using an electronic pedometer (Kenz Lifecorder-GS, Suzuken Co., Ltd., Nagoya, Japan). Under laboratory conditions, this instrument can determine step counts with intra-model reliability of 0.998 and accuracy within $3 \%$ of the actual number of steps taken. ${ }^{17}$ After providing their informed consent, the patients wore the device on the side of their waist. ${ }^{18}$ We instructed patients to wear the device for as long as possible, from when they change clothes in the morning until they change into nightclothes. The device recorded the daily steps and intensity of physical activity every $4 \mathrm{~s}$ throughout the day. The data, including the number of daily steps, intensity of physical activity, and time during which the device was worn, were collected during regular visits to the outpatient department, pretreatment and T2. Days on which the device was worn for less than five hours were excluded from the analysis. ${ }^{14,19,20}$ Daily steps and intensity of physical activity were monitored throughout the study period until the cutoff date. Physical activity which included three or more metabolic equivalents (METs) was defined as MVPA. ${ }^{21}$ The average number of daily steps and duration of MVPA for the seven days before the pretreatment assessment was set as the parameter for daily steps and duration of MVPA during the pretreatment period. Likewise, the average number of daily steps and duration of MVPA for the seven days before T2 was set as 
the parameter for $\mathrm{T} 2$. The change rate in daily steps from pretreatment to $\mathrm{T} 2$ was derived using the following formula:

$$
\left.\left[\begin{array}{l}
\text { changerateindailysteps }(\%)= \\
\left\{\begin{array}{l}
\text { (dailystepsatT } 2-\text { pretreatmentdailysteps }) \\
\text { /pretreatmentdailysteps }
\end{array}\right.
\end{array}\right\} \times 100\right]
$$

The patients were classified into two groups based on the change rate as follows: those with an equal or higher median change rate in daily steps were classified as active, while those with a lower median change rate in daily steps were classified as inactive.

\section{Assessment of Skeletal Muscle Mass}

Lumbar skeletal muscle mass was estimated by analyzing electronically stored computed tomography (CT) images using sliceOmatic software (version 5.0, TomoVision, Montreal, Quebec, Canada). The CT images were contrast-enhanced or unenhanced with a slice thickness of $5 \mathrm{~mm}$. Furthermore, two consecutive CT images at the third lumbar vertebra (L3) were selected to measure the skeletal muscle's crosssectional area identified based on the Hounsfield unit thresholds from -29 to +150 . The cross-sectional areas $\left(\mathrm{cm}^{2}\right)$ of the L3 region muscle were computed for each image. The mean value of two images was normalized for height in square meters and reported as lumbar skeletal muscle index. ${ }^{22}$

\section{Definition of Skeletal Muscle Depletion and Cancer Cachexia}

Skeletal muscle depletion was defined based on lumbar skeletal muscle index cutoffs of less than $43.0 \mathrm{~cm}^{2} / \mathrm{m}^{2}$ and $53.0 \mathrm{~cm}^{2} / \mathrm{m}^{2}$ for men with a body mass index of less than $25.0 \mathrm{~kg} / \mathrm{m}^{2}$ and $25.0 \mathrm{~kg} / \mathrm{m}^{2}$ or higher, respectively, and the cutoff was $41.0 \mathrm{~cm}^{2} / \mathrm{m}^{2}$ for women. ${ }^{23}$ According to the consensus criteria, we defined cancer cachexia as an unintentional weight loss of higher than $5 \%$ in the preceding six months, weight loss of higher than $2 \%$ in patients with a body mass index of less than $20.0 \mathrm{~kg} / \mathrm{m}^{2}$, or the presence of skeletal muscle depletion. ${ }^{24}$ The patients' weight six months before enrolling in the study were obtained through interviews with them and their family members before the study's commencement.

\section{Assessment of Physical Function}

An incremental shuttle walking test was conducted based on a recent guideline ${ }^{25}$ and an original protocol presented by Singh et al. ${ }^{26}$ A $10 \mathrm{~m}$ course was created in the hospital corridor. Walking speed was indicated by a timed signal played on a compact disk recorder provided by the manufacturer (Japanese version, the Graduate School of Biomedical Sciences, Nagasaki University, Japan, 2000). Assessments for all patients were conducted once under standardized conditions with careful observation to ensure that they did not exceed their exercise limits. An instructor accompanied the patients along the course during the assessments but did not interfere with the process by encouraging them. The assessment was concluded (1) by the patient when they were exhausted and unable to maintain the required walking speed, (2) by the instructor when the patient could not complete a shuttle within the allotted time (ie, walk for more than $0.5 \mathrm{~m}$ away from the cone within the set time), or (3) when the patient experienced $85 \%$ or higher heart rate than the predicted maximal heart rate derived using the formula $[210-(0.65 \times$ age $)]$. Incremental shuttle walking distance denoted the maximum walking distance.

Hand-grip strength was measured using a grip strength dynamometer (GRIP-D, Takei Scientific Instruments Co., LTD, Niigata, Japan). The device's handle was adjusted according to participants' hand size such that the index finger of each hand was at $90^{\circ}$ flexion between the proximal and middle phalangeal joint. Additionally, an instructor demonstrated the proper form-placing the feet hipwidth apart and holding the dynamometer away from the body and in line with the forearm at thigh level so that it did not touch the body, while ensuring that the arm was fully extended - and emphasized a quick and hard squeeze of the handle. ${ }^{27}$ Patients did not perform any practice tests. A trial was performed for each hand and the results from the hand yielding the highest force were used for this analysis.

\section{Disability-Free Survival and Overall Survival}

To assess daily living activities, the Barthel Index was estimated by the attending physician or physiotherapist for each hospital visit. The assessment interval was at least once in twelve weeks. The disability-free survival $(\mathrm{DFS})^{28}$ duration was calculated as the time between T2 and the onset of a disabling event. A disabling event is defined as a decrease in the Barthel Index from the pretreatment value by ten points or higher. The event was considered an actual event when the condition 
persisted for more than two weeks from the initial report. In confirmed events, the initial reports' dates were used as the event dates in the analysis. The overall survival duration was calculated as the time between $\mathrm{T} 2$ and death. The DFS and overall survival were censored on the final visit for patients whose disabling events or deaths were not confirmed.

\section{Assessment of Healthcare Resource Utilization}

Medical claims data, including the numbers of hospitalization and outpatient visits, length of hospital stays, healthcare resource utilization, and medical costs were obtained from hospital electronic medical records. ${ }^{12}$ Medical claims data were obtained through the local clinics and hospitals' institutional coordination offices for patients receiving medical care at another hospital. Certified medical accountants estimated inpatient medical costs. In this study, medical costs refer to the hospital's actual revenue from the health insurance funds of the Japanese healthcare system. We did not include medical costs for home care. For healthcare utilization analysis, visits (or hospitalizations) for supportive care included all visits (or hospitalizations) involving physical examinations, excluding visits (or hospitalizations) for chemotherapy, radiotherapy, or surgery.

\section{Statistical Analysis}

Since this was an exploratory study, the sample size was determined based on the feasibility of the study. Pretreatment patient characteristics of both groups were compared using Fisher's exact test for categorical variables and the Wilcoxon rank-sum test for continuous variables. The overall survival and DFS rates from a landmark point (ie, T2) were estimated using the Kaplan-Meier method and compared using a Log rank test. Cox proportional hazard models were used to estimate the association between the change in daily steps and overall survival or DFS. We used the mean cumulative function for the recurrent event analysis $^{29}$ of the cumulative length of hospital stays and medical costs related to cancer care from a landmark point, as performed in previous studies. ${ }^{12,30,31}$ For all analyses, the level of statistical significance was set at $p=0.05$. Statistical analyses were performed using JMP Pro 14.0.0 for Mac (SAS Institute Inc., USA).

\section{Results}

\section{Patient Characteristics}

Twenty-nine patients were enrolled in the study from February 27, 2014 to October 13, 2017. One patient discontinued participation for personal reasons, whereas seven patients did not undergo initial chemotherapy, opting for the best possible supportive care. Therefore, the remaining 21 patients were included in the analyses (Figure 1). The median period from the diagnosis of metastatic or postoperative recurrent disease to study enrolment was 1.9 weeks (range: 0.0-29.9 weeks). The median age was 75 years (range: 70-82 years; Table 1). Seventeen patients $(81.0 \%)$ had stage IV, and four $(19.0 \%)$ had postoperative recurrence. Seven and fourteen patients exhibited a PS of 0 and 1, respectively, and fifteen and six patients received cytotoxic and targeted regimens as the first-line chemotherapy, respectively. The median body mass index was $21.9 \mathrm{~kg} / \mathrm{m}^{2}$ (range: 19.0-28.1 kg/m²). Skeletal muscle depletion and cancer cachexia were diagnosed in fourteen $(66.7 \%)$ and thirteen $(61.9 \%)$ patients. The median incremental shuttle walking distance was $360 \mathrm{~m}$ (range: $140-550 \mathrm{~m}$ ) in men and 355m (range: $120-480 \mathrm{~m}$ ) in women. The median hand-grip strength was $30.6 \mathrm{~kg}$ (range: $25.6-37.2 \mathrm{~kg}$ ) in men and $23.7 \mathrm{~kg}$ (range: $19.0-30.3 \mathrm{~kg}$ ) in women. The median number of daily steps and duration of MVPA at pretreatment was 3389.6 steps (range: $1141.6-17,711.3$ steps) and $4.4 \mathrm{~min}-$ utes (range: 0-60.9 minutes), respectively.

\section{Change in Physical Activity}

The median change rate in daily steps from pretreatment to $\mathrm{T} 2$ was $-12.5 \%$ (range: -89.5 to $+91.7 \%$ ) in all patients (Table 2 ). Furthermore, eleven and ten patients were classified as active and inactive, respectively. The median change in daily steps was +490 steps $(+6.4 \%)$ and -1333 steps $(-35.6 \%)$ for active and inactive patients, respectively. The duration of MVPA at T2 was 2.3 minutes (range: 0.0-52.6 minutes), and the median change rate in the duration of MVPA from pretreatment to T2 was $-24.3 \%$ (range: -100.0 to $45.5 \%$ ) in all patients (Table 2).

\section{Differences in Patient Characteristics}

The pretreatment lumbar skeletal muscle index among men was significantly lower for active patients than for inactive patients (39.4 vs $45.2 \mathrm{~cm}^{2} / \mathrm{m}^{2}, p<0.05$, Table 1 ). No significant difference was noted between the groups in terms of pretreatment PS, chemotherapy regimen types, the incidence of skeletal muscle depletion, cancer 
First study enrollment

(Feb 27, 2014)

Last study enrollment

(Oct 13, 2017)
At one year from the landmark point (T2)

Data cutoff date (Aug 31, 2018)

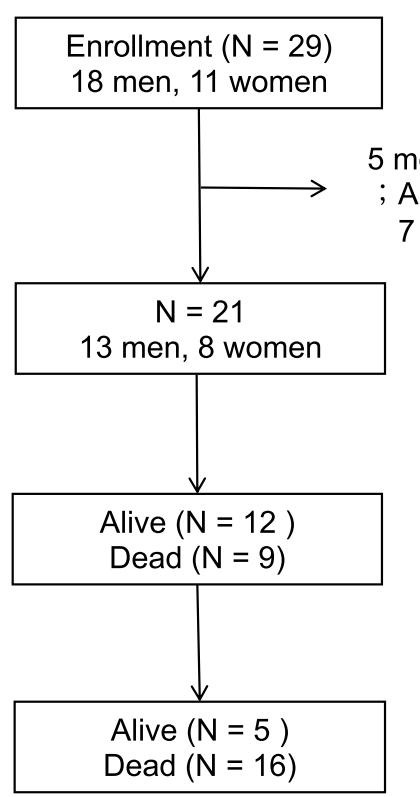

21 patients were evaluable for the analysis for length of hospital stays and medical costs

All 29 patients were evaluable for the baseline assessment

5 men and 3 women excluded

A patient wanted to decline participation in the study

7 patients did not receive chemotherapy

21 patients were evaluable for the analysis for disability-free and overall survival

Figure I Patient flowchart. Flow diagram of patient recruitment and follow-up.

cachexia, and physical function. Objective tumor response was observed in four cases $(40.0 \%)$ for inactive patients and four cases $(36.4 \%)$ for active patients without statistically significant differences.

\section{Overall Survival and Disability-Free Survival}

Of the 21 patients, sixteen (76.2\%) had a disability and passed away by the cutoff date. The median follow-up period was 18.7 months (95\% confidence interval [CI], 15.1-21.8). Inactive patients exhibited significantly shorter overall survival than active patients (8.8 vs 20.4 months, $p<0.05$; Figure 2A). The one-year survival rates were $40.0 \%$ in inactive patients and $72.7 \%$ in active patients. Inactive patients also exhibited significantly shorter DFS than active patients (6.4 vs 19.9 months, $p<0.05$; Figure 2B). The one-year DFS rates were $20.0 \%$ in inactive patients and $72.7 \%$ in active patients. The hazard ratio for overall survival in inactive patients was 1.92 (95\% CI, 0.59-7.07) adjusted for known prognostic factors ${ }^{32,33}$ including PS and the presence of mutations in the epidermal growth factor receptor (EGFR). The hazard ratio for DFS in inactive patients was 2.17 (95\% CI, 0.66-8.2) after adjustment for PS and EGFR mutation. In the subset analyses of patients who had received cytotoxic chemotherapy $(\mathrm{n}=15)$, similar differences between groups were observed regarding overall survival and DFS
(Supplemental Figure 1). In addition, the patients were divided into two groups based on their median MVPA duration at pretreatment (4.4 minutes per day). Furthermore, overall survival and DFS were compared between the two groups and there was no significant difference between the two groups. (Supplemental Figure 2).

\section{Hospital Stays and Medical Costs}

The cumulative length of hospital stays for the first year tended to be longer for inactive patients than active patients (23.7 vs 6.3 days/person/year), and the difference continued to widen over the available follow-up period (Figure 3A). Moreover, inactive patients tended to require more frequent hospitalizations (1.9 vs 0.8 times/person/year) and unplanned outpatient visits or emergency hospitalizations (1.7 vs 0.9 times/person/year) than active patients (Table 3). Conversely, cumulative medical costs for the first year were similar for inactive and active patients ( $¥ 3.0$ vs $¥ 2.5 \mathrm{million} /$ person/year [US\$30,000 vs US\$25,000/ person/year], Table 3); however, the curves of cumulative medical costs of both groups were different in the second year and continued to diverge over the available follow-up period (Figure 3B). For the breakdown of healthcare utilization in the first year, active patients had higher expenses for outpatient care (¥2.2 vs $¥ 1.4 \mathrm{million} /$ person/year [US\$22,000 vs US\$14,000/ 
Table I Baseline Patient Characteristics

\begin{tabular}{|c|c|c|c|c|}
\hline & All $n=21$ & Active $^{d} n=11$ & Inactive ${ }^{e} n=10$ & p-value \\
\hline Age, median (range) & $75(70-82)$ & $73(70-81)$ & $76(72-82)$ & NS \\
\hline Gender (Women: Men) & 8: 13 & 6: 5 & 2: 8 & NS \\
\hline Stage, n (\%) & & & & NS \\
\hline Stage IV & $17(81.0)$ & $9(8 \mid .8)$ & $8(80.0)$ & \\
\hline Postoperative recurrence & $4(19.0)$ & $2(18.2)$ & $2(20.0)$ & \\
\hline PS, n (\%) & & & & NS \\
\hline 0 & $7(33.3)$ & $5(45.5)$ & $2(20.0)$ & \\
\hline 1 & $14(66.7)$ & $6(54.5)$ & $8(80.0)$ & \\
\hline Treatment, n (\%) & & & & NS \\
\hline Cytotoxic regimen & $15(7 \mid .4)$ & $6(54.5)$ & $9(90.0)$ & \\
\hline Targeted regimen & $6(28.6)$ & $5(45.5)$ & I (I0.0) & \\
\hline Never smoke, n (\%) & $8(38.1)$ & $5(45.5)$ & $3(30.0)$ & NS \\
\hline Comorbidities, n (\%) & & & & NS \\
\hline Cardiovascular disease & $6(28.6)$ & $2(18.2)$ & $4(40.0)$ & \\
\hline Cerebrovascular disease & I (4.8) & I (9.I) & $0(0.0)$ & \\
\hline Pulmonary disease & $2(9.5)$ & $2(18.2)$ & $0(0.0)$ & \\
\hline Bone and joint disorder & $6(28.6)$ & I (9.I) & $5(50.0)$ & \\
\hline Type 2 diabetes & $5(23.8)$ & $2(18.2)$ & $3(30.0)$ & \\
\hline Other cancer & $2(9.5)$ & I (9.I) & $I(10.0)$ & \\
\hline BMI $\left(\mathrm{kg} / \mathrm{m}^{2}\right)$, median (range) & $21.9(19.0-28.1)$ & $21.9(19.0-28.1)$ & $21.6(19.2-25.2)$ & NS \\
\hline \multicolumn{5}{|l|}{ LSMI $\left(\mathrm{cm} / \mathrm{m}^{2}\right)$, median (range) } \\
\hline Women & $36.7(31.5-46.2)$ & $36.1(31.5-46.2)$ & $36.7(36.5-37.0)$ & NS \\
\hline Men & $43.9(38.2-50.6)$ & $39.4(38.2-45.1)$ & $45.2(39.9-50.6)$ & $<0.05$ \\
\hline Skeletal muscle depletion, $\mathrm{n}(\%)^{\mathrm{a}}$ & $14(66.7)$ & $9(81.8)$ & $5(50.0)$ & NS \\
\hline Cachexia, n (\%) ${ }^{b}$ & $13(61.9)$ & $6(54.5)$ & $7(70.0)$ & NS \\
\hline \multicolumn{5}{|l|}{ ISWD (m), median (range) } \\
\hline Women & $355(120-480)$ & $355(120-480)$ & $335(270-400)$ & NS \\
\hline Men & $360(140-550)$ & $340(270-550)$ & $370(140-460)$ & NS \\
\hline \multicolumn{5}{|l|}{ HGS (kg), median (range) } \\
\hline Women & $23.7(19.0-30.3)$ & $21.4(19.0-26.1)$ & $28.1(25.9-30.3)$ & NS \\
\hline Men & $30.6(25.6-37.2)$ & $29.2(25.6-37.2)$ & $30.8(25.8-34.9)$ & NS \\
\hline Daily steps, median (range) & $3389.6(|14| .6-|7,7| 1.3)$ & $3034.4(1141.6-17,7 \mid 1.3)$ & $4638.8(1297.6-5824.1)$ & NS \\
\hline MVPA $^{c}$ (minutes), median (range) & $4.4(0-60.9)$ & $4.3(1-60.9)$ & $5.2(0-13.3)$ & NS \\
\hline
\end{tabular}

Notes: aSkeletal muscle depletion was defined based on LSMI cutoffs of less than $43.0 \mathrm{~cm}^{2} / \mathrm{m}^{2}$ and $53.0 \mathrm{~cm}^{2} / \mathrm{m}^{2}$ for men with a BMI of less than $25.0 \mathrm{~kg} / \mathrm{m}^{2}$ and $25.0 \mathrm{~kg} / \mathrm{m}^{2}$ or higher, respectively, and the cutoff was $41.0 \mathrm{~cm}^{2} / \mathrm{m}^{2}$ for women. ${ }^{\mathrm{b}}$ Cancer cachexia was defined as the unintentional weight loss of higher than $5 \%$ in the preceding six months, weight loss of higher than $2 \%$ in patients with a BMI of less than $20.0 \mathrm{~kg} / \mathrm{m}^{2}$, or the presence of skeletal muscle depletion. ${ }^{\mathrm{C}}$ MVPA was defined as physical activity of 3 metabolic equivalents (METs) or more. ${ }^{\mathrm{d}}$ Active was defined as a patient group with the change rate in daily steps from pretreatment to T2 of $-12.5 \%$ or higher. ${ }^{\mathrm{e}}$ Inactive was defined as a patient group with the change rate in daily steps from pretreatment to T2 of less than $-12.5 \%$. Significant difference $(P<0.05)$ tested by Fisher's exact tests or Wilcoxon rank-sum test.

Abbreviations: PS, eastern cooperative oncology group performance status; BMI, body mass index; LSMI, lumbar skeletal muscle index; ISWD, incremental shuttle walking distance; HGS, hand-grip strength; MVPA, moderate to vigorous physical activity. 
Table 2 Changes in Physical Activity from Pretreatment to T2

\begin{tabular}{|l|l|l|}
\hline & \multicolumn{1}{|c|}{ Pretreatment } & \multicolumn{1}{|c|}{ T2 $^{\mathbf{b}}$} \\
\hline Daily steps, median (range) & $3389.6(1141.6-17,711.3)$ & $3398.6(345.1-18,847.3)$ \\
\hline MVPA $^{\text {a }}$ (minutes), median (range) & $4.4(0-60.9)$ & $2.3(0-52.6)$ \\
\hline
\end{tabular}

Notes: aMVPA was defined as physical activity of 3 metabolic equivalents (METs) or more. ${ }^{\text {b}} \mathrm{T} 2$ was $12 \pm 4$ weeks from enrolment.

Abbreviation: MVPA, moderate to vigorous physical activity.

person/year], $p<0.05)$ and chemotherapy (¥2.0 vs $¥ 0.8 \mathrm{million} /$ person/year [US\$20,000 vs US\$8000/person/year], $p<0.05)$ than inactive patients. In comparison, inactive patients had higher costs for inpatient care ( $¥ 1.6$ vs $¥ 0.3 \mathrm{million} /$ person/year [US\$16,000 vs US\$3000/person/year]), palliative radiation therapy or operation (¥1.4 vs ¥0.1 million/person/year [US $\$ 14,000$ vs US\$1000/person/year]), and supportive care (¥0.7 vs ¥0.4 million/person/year [US\$7000 vs US\$4000/person/year]). The difference was not statistically significant.

A

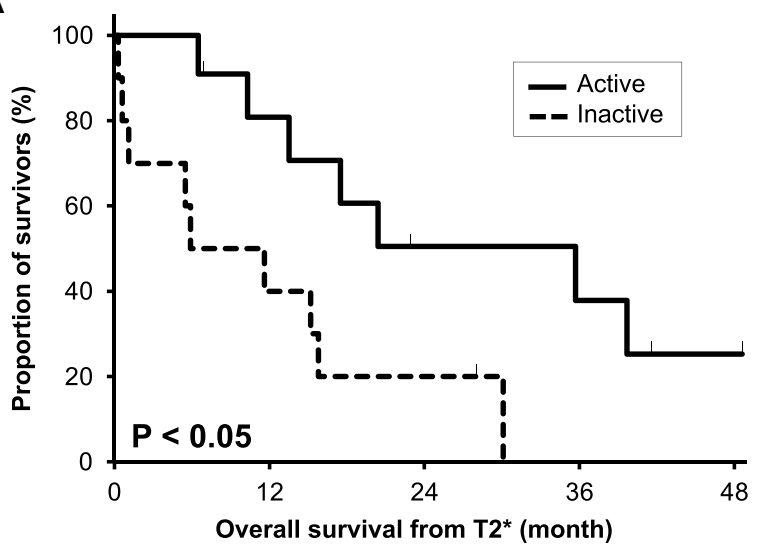

\section{Discussion}

To the best of our knowledge, this study is the first attempt to evaluate the influence of physical inactivity during initial chemotherapy on the risk of disability and hospitalization in later life among older patients with advanced NSCLC. First, we found that most of our patients experienced decreased physical activity, with a median decrease of $12.5 \%$ in daily steps and $24.3 \%$ in the duration of MVPA. Second, active patients lived longer without disability than inactive patients. Finally, inactive patients required long and frequent inpatient care and spent less on outpatient chemotherapy than active patients.

B

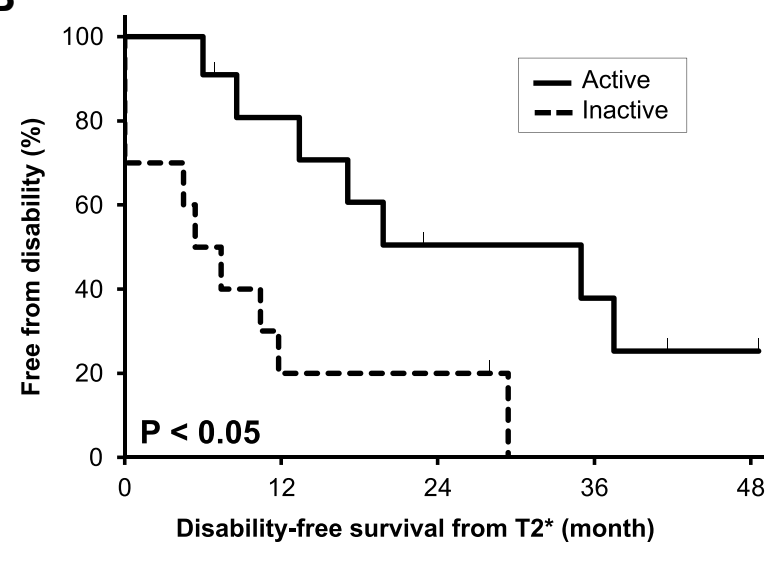

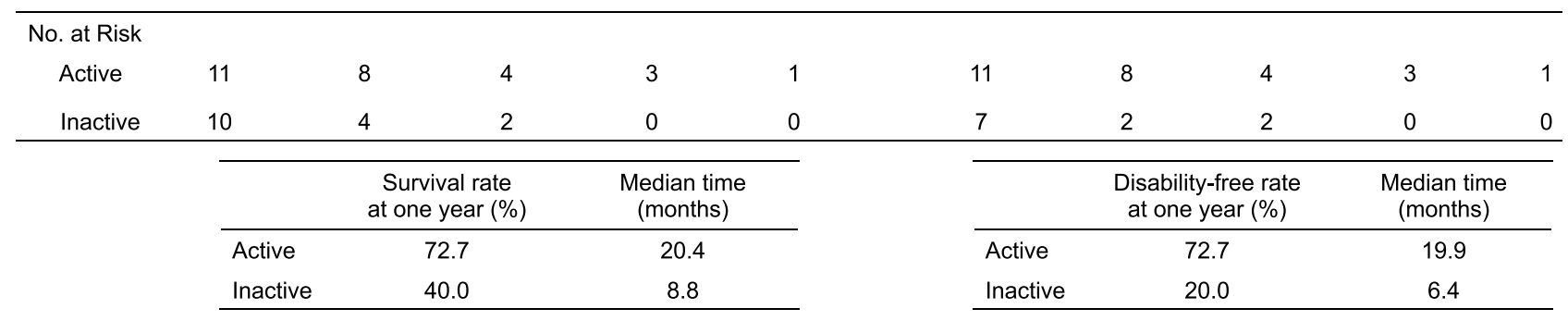

Figure 2 Overall and disability-free survival curves. Panel (A) Kaplan-Meier curve of overall survival from T2. Panel (B) Kaplan-Meier curve of disability-free survival from T2. A solid line indicates active patients and a dotted line indicates inactive patients. P-values were calculated using Log rank tests. A disabling event is defined as a decrease in Barthel Index from the pretreatment value by 10 points or higher. The overall and disability-free survival was censored on the final visit for patients whose deaths or disabling events were not confirmed. $* T 2$ was $12 \pm 4$ weeks from enrolment. 
A Mean cumulative length of hospital stays

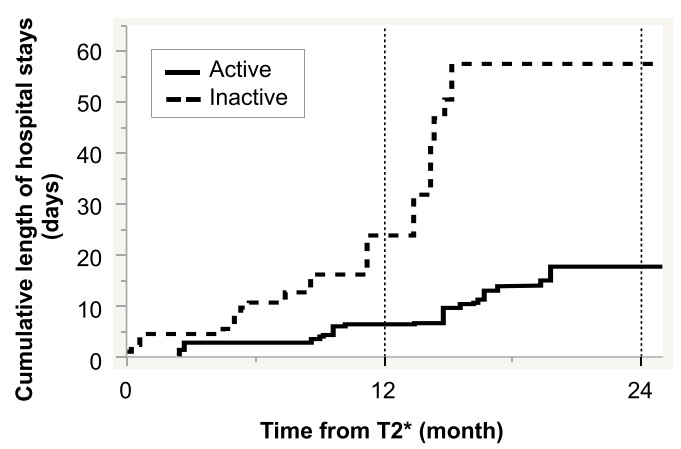

B Mean cumulative medical costs

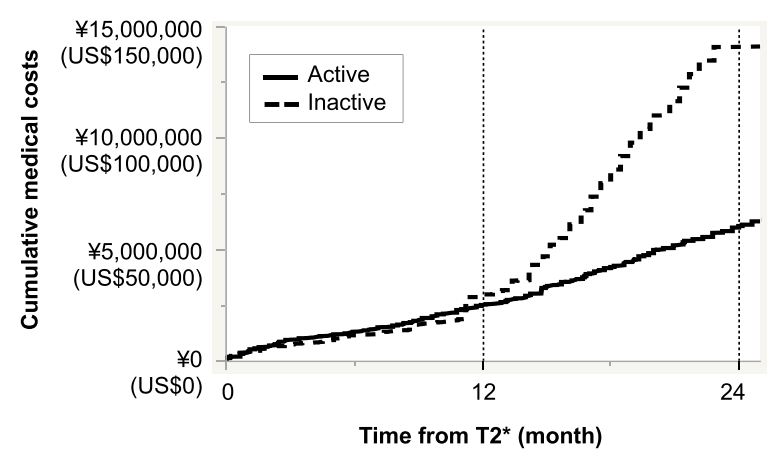

Figure 3 Difference between active and inactive patients in terms of cumulative length of hospital stays and medical costs from T2. Curves of cumulative functions for the length of hospital stays panel $(\mathbf{A})$ and medical costs panel $(\mathbf{B})$ in active patients (solid line) and inactive patients (dotted line). $*$ T2 was $12 \pm 4$ weeks from enrolment.

Patients with lung cancer have been reported to have decreased physical activity than healthy adults throughout the cancer trajectory. ${ }^{34}$ Specifically, in patients with advanced NSCLC, physical inactivity has been shown to affect prognosis negatively. ${ }^{35,36}$ However, few studies have focused on physical inactivity in the early phase of the cancer trajectory. Our study indicated that physical inactivity during initial chemotherapy may deteriorate the overall survival and DFS of older patients with advanced NSCLC. Their behavior during treatment may be more important, for better long-term outcomes, than their physical condition before treatment. Although the multivariate analysis' results demonstrated that physical inactivity was not an independent prognostic factor for overall survival and DFS, this may have been a false negative due to the small sample size, since the $95 \%$ CI for overall survival and DFS was wide.
The use of medical resources by patients with cancer has been reported to increase with the presence of sarcopenia, ${ }^{37}$ cachexia, ${ }^{12}$ frailty, ${ }^{38}$ and unsatisfactory PS. ${ }^{39}$ Several studies have reported the association between physical inactivity and medical resources in patients with cancer. ${ }^{36,40}$ As per the present study results, inactive patients required more extended hospital stays and had higher costs than active patients. Moreover, medical treatments were considerably different between active and inactive patients. Although the total annual costs were similar in the first year, most medical expenses in active patients were related to outpatient chemotherapy, whereas, for inactive patients, these were related to inpatient supportive care.

This study has several limitations. First, this was an observational prospective study with a small number of

Table 3 Differences in Socioeconomic Parameters of Cancer Treatment in the First Year

\begin{tabular}{|c|c|c|c|}
\hline Socioeconomic Parameters for the First Year ${ }^{a}$ & Active & Inactive & p-value \\
\hline Cumulative no. of the length of hospital stays ${ }^{b}$ & $6.3 \pm 3.7$ & $23.7 \pm 10.1$ & NS \\
\hline Cumulative times of hospitalization ${ }^{c}$ & $0.8 \pm 0.5$ & $1.9 \pm 0.7$ & NS \\
\hline Cumulative no. of unplanned visits or emergency hospitalizations ${ }^{c}$ & $0.9 \pm 0.4$ & $1.7 \pm 0.5$ & NS \\
\hline \multicolumn{4}{|l|}{ Cumulative medical costs ${ }^{d}$} \\
\hline Total & $2.5 \pm 0.4$ & $3.0 \pm 1.0$ & NS \\
\hline Outpatient care & $2.2 \pm 0.3$ & $1.4 \pm 0.2$ & $<0.05$ \\
\hline Inpatient care & $0.3 \pm 0.2$ & $1.6 \pm 0.9$ & NS \\
\hline \multicolumn{4}{|l|}{ Cumulative costs for resource utilization ${ }^{d}$} \\
\hline Chemotherapy & $2.0 \pm 0.3$ & $0.8 \pm 0.3$ & $<0.05$ \\
\hline Radiotherapy/Operation & $0.1 \pm 0.1$ & $1.4 \pm 0.9$ & NS \\
\hline Supportive care & $0.4 \pm 0.2$ & $0.7 \pm 0.2$ & NS \\
\hline
\end{tabular}

Notes: ${ }^{a} \mathrm{~A}$ mean cumulative function was used for recurrent event analysis of cumulative length of hospital stays and medical costs related to cancer care for the first year from T2. T2 was $12 \pm 4$ weeks from enrolment. ${ }^{b}$ Days per person. ${ }^{C}$ Times per person. ${ }^{d} \times 10^{6} \mathrm{JP}$ yen per person $\left(\times 10^{4}\right.$ US $\$$ per person). 
participants, and, therefore, it may not have had sufficient power to draw definite conclusions. In addition, because this study included only Japanese patients who received treatment, our results may not be generalizable to all older patients with advanced NSCLC in other health care settings. Second, the chemotherapy administered during the study included different types of regimens, which possibly influenced the measured outcomes. Third, the health insurance system in Japan is different from those in other countries. Moreover, the standard of care and the medical environment is rapidly changing with advances in medicine. Thus, our results cannot be generalized to other populations in differing medical situations across countries. Fourth, since this study evaluated daily steps using only a pedometer, physical activity assessment may not be completely accurate. While a pedometer is an objective method of assessing daily steps, wearing it on the waist may have underestimated upper body movements and activities such as cycling, swimming, and resistance exercise. $^{41}$ Therefore, the simultaneous use of a pedometer and a questionnaire such as the Physical Activity Scale for the Elderly (PASE) ${ }^{42}$ is recommended to assess daily physical activity in patients with cancer. ${ }^{41}$ Finally, biases such as those involved in selection and measurement could not be avoided. However, there are few previous reports on changes in physical activity over time in older patients with advanced NSCLC, and the results of this study are valuable in demonstrating the importance of maintaining physical activity in these patients during treatment.

The vulnerability of older patients with advanced lung cancer is influenced by multiple factors and requires a multidisciplinary team approach rather than a single intervention. ${ }^{15}$ Since exercise is one of the most critical interventions for the frail older population, ${ }^{43}$ it must be incorporated into the supportive program for older patients with advanced cancer. The disease's status and treatmentrelated side effects are major factors that inhibit cancer patients from participating in physical activities. ${ }^{44}$ However, physical activity has been reported to improve cardiorespiratory fitness, muscular strength, body composition, quality of life, anxiety, and depression, and help mitigate treatment-related side effects such as nausea, vomiting, peripheral neuropathy, fatigue, arthralgia, and myalgia. ${ }^{45} \mathrm{~A}$ recent systematic review reported that exercise appears to be an effective intervention for improving physical function, quality of life, fatigue, body composition, psychosocial function, and sleep quality in patients with advanced cancer. ${ }^{46}$ Due to the physical vulnerability of patients with advanced cancer, even an increase in light physical activity may foster their ability to be more functional and active in everyday life. ${ }^{47}$ The importance of promoting physical activity using pedometers or accelerometers has been recognized in geriatric medicine ${ }^{48}$ and walking is classified as a low-intensity exercise intervention for older patients with cancer. Monitoring physical activity, using devices, can positively impact numerous clinically meaningful outcomes in cancer patients. ${ }^{49}$ In addition to using equipment to promote physical activity, multidisciplinary promotive counseling is needed for positive behavioral change in physical activity. ${ }^{20,44}$

Naito et al recently reported the feasibility of a regimen combining exercise and nutritional interventions for older Japanese patients with advanced cancer. ${ }^{19,20}$ Their exercise prescription combined promotive counseling for physical activity with homebased resistance training and nutritional advice by multidisciplinary team, which was initiated just after diagnosing the advanced pancreatic or NSCLC. Exploratory analysis showed the preservation of physical function, muscle mass, and daily steps during the study period. The intervention is being tested in an ongoing randomized controlled trial (trial registration number: UMIN000028801). ${ }^{50}$ The current results support early physical activity intervention by multidisciplinary team, which is aimed not only at maintaining physical function, but also improving long-term outcomes, including disability, medical dependency, and costeffectiveness in the care of older patients with advanced cancer.

\section{Conclusion}

Physical inactivity during initial chemotherapy may be a risk factor for developing disabilities and requiring hospitalization in later life for older patients with advanced NSCLC. Our findings may indicate the need for lifestyle interventions with multidisciplinary teams, which include physicians, nurses, and physiotherapists, for older patients with advanced lung cancer during active cancer treatment. A large-samplesized study is needed to validate our findings.

\section{Abbreviations}

NSCLC, non-small-cell lung cancer; PS, eastern Cooperative Oncology Group performance status; MVPA, moderate to vigorous physical activity; METs, metabolic 
equivalents; CT, computed tomography; DFS, disability-free survival; EGFR, epidermal growth factor receptor.

\section{Data Sharing Statement}

The datasets used and/or analyzed during the current study are available from the corresponding author upon reasonable request.

\section{Ethics Approval and Informed Consent}

The study was approved by the Shizuoka Cancer Center's institutional review board (Shizuoka, Japan) and registered on the University Hospital Medical Information Network Clinical Trials Registry in Japan (trial registration number: UMIN000012845). All patients provided written informed consent. All assessments were carried out under the ethical principles outlined in the Declaration of Helsinki. There were no major changes in the study methods after trial commencement.

\section{Consent for Publication}

All authors and their affiliations consented to publication of the study in this journal.

\section{Acknowledgments}

We would like to thank Ms. Asami Oba, a certified medical accountant at Shizuoka Cancer Center, for contributing to medical costs analyses.

\section{Author Contributions}

All authors made substantial contributions to conception and design, acquisition of data, or analysis and interpretation of data; took part in drafting the article or revising it critically for important intellectual content; agreed to submit to the current journal; gave final approval of the version to be published; and agree to be accountable for all aspects of the work.

\section{Funding}

The 35th grant-in-aid from the Japanese Foundation for the Multidisciplinary Treatment of Cancer in 2014 supported this work.

\section{Disclosure}

Mr. Yonenaga has nothing to disclose. Dr. Naito reports personal fees from Ono Pharmaceutical Co., Ltd., and Mochida Pharmaceutical Co., Ltd., which are unrelated to the submitted work. Mr. Okayama has nothing to disclose.
Ms. Kitagawa has nothing to disclose. Ms. Mitsuhashi has nothing to disclose. Mr. Ishii has nothing to disclose. Dr. Fuseya has nothing to disclose. Ms. Inano has nothing to disclose. Mr. Morikawa has nothing to disclose. Ms. Sugiyama has nothing to disclose. Mr. Mori has nothing to disclose. Mr. Notsu has nothing to disclose. Mr. Kawabata has nothing to disclose. Dr Akira Ono reports honoraria from AstraZeneca, Chugai, Ono Pharmaceutical, and MSD, outside the submitted work. Dr. Kenmotsu reports personal fees from Ono Pharmaceutical Co., Ltd., Eli Lilly K.K., Kyowa Hakko Kirin Co., Ltd., Bristol-Myers Squibb Company, MSD K.K., Novartis Pharma K.K., and grants from DaiichiSankyo Co., Ltd., and grants and personal fees from Chugai Pharmaceutical Co., Ltd., Boehringer Ingelheim Company, AstraZeneca K.K., Pfizer, and Taiho Pharma, which are unrelated to the submitted work. Dr. Murakami reports personal fees from Bristol-Myers Squibb Company, Ono Pharmaceutical Co., Ltd., MSD K.K., and grants from AbbVie Company, Daiichi-Sankyo Co., Ltd., IQvia Company, and grants and personal fees from AstraZeneca K.K., Chugai Pharmaceutical Co., Ltd., Eli Lilly K.K., Pfizer, Novartis, Taiho Pharmaceutical Co., Ltd., Takeda Pharmaceutical Co., Ltd., which are unrelated to the submitted work. Dr. Tanuma has nothing to disclose. Dr. Takahashi reports grants from Japan Agency for Medical Research and Development during the conduct of the study, and personal fees from Boehringer Ingelheim Company, Roche Diagnostics K.K., and grants and personal fees from AstraZeneca K.K., Chugai Pharmaceutical Co., LTD., Eli Lilly K.K., Ono Pharmaceutical Co., Ltd., MSD K.K., Pfizer Japan Inc., which are unrelated to the submitted work. The authors report no other conflicts of interest in this work.

\section{References}

1. Takayuki N, Keiko T, Junji U, et al. Advanced non-small-cell lung cancer in elderly patients: patient features and therapeutic management. Biomed Res Int. 2018;30:8202971.

2. Zappa C, Mousa SA. Non-small cell lung cancer: current treatment and future advances. Transl Lung Cancer Res. 2016;5(3):288-300. doi:10.21037/tlcr.2016.06.07

3. Presley CJ, Reynolds CH, Langer CJ. Caring for the older population with advanced lung cancer. Am Soc Clin Oncol Educ Book. 2017;37 (37):587-596. doi:10.1200/EDBK_179850

4. Ministry of Health, Labour and Welfare [homepage on the internet]. Estimates of medical care expenditure, summary for 2018. Available from: https://www.mhlw.go.jp/toukei/saikin/hw/k-iryohi/18/index. html. Accessed January 3, 2021.

5. Cruz-Jentoft AJ, Bahat G, Bauer J, et al; for European Working Group on Sarcopenia in Older People 2 (EWGSOP2), and the Extended Group for EWGSOP2. Sarcopenia: revised European consensus on definition and diagnosis. Age Ageing. 2019;48(4):601. doi:10.1093/ageing/afz046 
6. Sattar S, Alibhai SM, Spoelstra SL, Fazelzad R, Puts MT. Falls in older adults with cancer: a systematic review of prevalence, injurious falls, and impact on cancer treatment. Support Care Cancer. 2016;24 (10):4459-4469. doi:10.1007/s00520-016-3342-8

7. Cruz-Jentoft AJ, Baeyens JP, Bauer JM, et al.; for European Working Group on Sarcopenia in Older People. Sarcopenia: European consensus on definition and diagnosis: report of the European Working Group on sarcopenia in older people. Age Ageing. 2010;39 (4):412-423. doi:10.1093/ageing/afq034

8. Asmis TR, Ding K, Seymour L, et al.; for National Cancer Institute of Canada Clinical Trials Group. Age and comorbidity as independent prognostic factors in the treatment of non small-cell lung cancer: a review of national cancer institute of Canada clinical trials group trials. J Clin Oncol. 2008;26(1):54-59. doi:10.1200/JCO.2007.12.8322

9. Gioulbasanis I, Georgoulias P, Vlachostergios PJ, et al. Mini Nutritional Assessment (MNA) and biochemical markers of cachexia in metastatic lung cancer patients: interrelations and associations with prognosis. Lung Cancer. 2011;74(3):516-520. doi:10.1016/j. lungcan.2011.05.009

10. Baracos VE, Reiman T, Mourtzakis M, Gioulbasanis I, Antoun S. Body composition in patients with non-small cell lung cancer: a contemporary view of cancer cachexia with the use of computed tomography image analysis. Am J Clin Nutr. 2010;91(4):1133S1137S. doi:10.3945/ajen.2010.28608C

11. Kimura M, Naito T, Kenmotsu H, et al. Prognostic impact of cancer cachexia in patients with advanced non-small cell lung cancer. Support Care Cancer. 2015;23(6):1699-1708. doi:10.1007/s00520014-2534-3

12. Naito T, Okayama T, Aoyama T, et al. Unfavorable impact of cancer cachexia on activity of daily living and need for inpatient care in elderly patients with advanced non-small-cell lung cancer in Japan: a Prospective Longitudinal Observational Study. BMC Cancer. 2017;17(1):800. doi:10.1186/s12885-017-3795-2

13. Lowe SS, Danielson B, Beaumont C, Watanabe SM, Baracos VE, Courneya KS. Associations between objectively measured physical activity and quality of life in cancer patients with brain metastases $J$ Pain Symptom Manage. 2014;48(3):322-332. doi:10.1016/j. jpainsymman.2013.10.012

14. Morikawa A, Naito T, Sugiyama M, et al. Impact of cancer cachexia on hospitalization-associated physical inactivity in elderly patients with advanced non-small-cell lung cancer. Asia Pac J Oncol Nurs. 2018;5(4):377-382. doi:10.4103/apjon.apjon_20_18

15. Fearon KC. Cancer cachexia: developing multimodal therapy for a multidimensional problem. Eur J Cancer. 2008;44(8):1124-1132. doi:10.1016/j.ejca.2008.02.033

16. Eisenhauer EA, Therasse P, Bogaerts J, et al. New response evaluation criteria in solid tumours: revised RECIST guideline (version 1.1). Eur $J$ Cancer. 2009;45(2):228-247. doi:10.1016/j. ejca.2008.10.026

17. Kumahara H, Schutz Y, Ayabe M, et al. The use of uniaxial accelerometry for the assessment of physical-activity-related energy expenditure: a validation study against whole-body indirect calorimetry. $B r \quad J \quad$ Nutr. 2004;91(2):235-243. doi:10.1079/ BJN20031033

18. Yasunaga A, Togo F, Watanabe E, et al. Sex, age, season, and habitual physical activity of older Japanese: the Nakanojo Study. $J$ Aging Phys Act. 2008;16(1):3-13. doi:10.1123/japa.16.1.3

19. Naito T, Mitsunaga S, Miura S, et al. Feasibility of early multimodal interventions for elderly patients with advanced pancreatic and non-small-cell lung cancer. J Cachexia Sarcopenia Muscle. 2019;10 (1):73-83. doi:10.1002/jcsm.12351

20. Mouri T, Naito T, Morikawa A, et al. Promotion of behavioral change and the impact on quality of life in elderly patients with advanced cancer: a physical activity intervention of the multimodal nutrition and exercise treatment for advanced cancer program. Asia Pac J Oncol Nurs. 2018;5(4):383-390. doi:10.4103/apjon.apjon_21_18
21. Pate RR, Pratt M, Blair SN, et al. Physical activity and public health. A recommendation from the centers for disease control and prevention and the american college of sports medicine. JAMA. 1995;273 (5):402-407. doi:10.1001/jama.1995.03520290054029

22. Mourtzakis M, Prado CM, Lieffers JR, Reiman T, McCargar LJ, Baracos VE. A practical and precise approach to quantification of body composition in cancer patients using computed tomography images acquired during routine care. Appl Physiol Nutr Metab. 2008;33(5):997-1006. doi:10.1139/H08-075

23. Martin L, Birdsell L, Macdonald N, et al. Cancer cachexia in the age of obesity: skeletal muscle depletion is a powerful prognostic factor, independent of body mass index. J Clin Oncol. 2013;31 (12):1539-1547. doi:10.1200/JCO.2012.45.2722

24. Fearon K, Strasser F, Anker SD, et al. Definition and classification of cancer cachexia: an international consensus. Lancet Oncol. 2011;12 (5):489-495. doi:10.1016/S1470-2045(10)70218-7

25. Singh SJ, Puhan MA, Andrianopoulos V, et al. An official systematic review of the European Respiratory Society/American Thoracic Society: measurement properties of field walking tests in chronic respiratory disease. Eur Respir J. 2014;44(6):1447-1478. doi:10.1183/09031936.00150414

26. Singh SJ, Morgan MD, Scott S, Walters D, Hardman AE. Development of a shuttle walking test of disability in patients with chronic airways obstruction. Thorax. 1992;47(12):1019-1024. doi:10.1136/thx.47.12.1019

27. Perna FM, Coa K, Troiano RP, et al. Muscular grip strength estimates of the U.S. population from the national health and nutrition examination survey 2011-2012. J Strength Cond Res. 2016;30 (3):867-874. doi:10.1519/JSC.0000000000001104

28. Naito T. Evaluation of the true endpoint of clinical trials for cancer cachexia. Asia Pac J Oncol Nurs. 2019;6(3):227-233. doi:10.4103/ apjon.apjon_68_18

29. Nelson W. Confidence limits for recurrence data: applied to cost or number of product repairs. Technometrics. 1995;37(2):147-157.

30. Humphries KH, Pu A, Gao M, Carere RG, Pilote L. Angina with "normal" coronary arteries: sex differences in outcomes. Am Heart J. 2008;155(2):375-381. doi:10.1016/j.ahj.2007.10.019

31. Smedira NG, Hoercher KJ, Lima B, et al. Unplanned hospital readmissions after HeartMate II implantation: frequency, risk factors, and impact on resource use and survival. JACC Heart Fail. 2013;1 (1):31-39. doi:10.1016/j.jchf.2012.11.001

32. Maemondo M, Inoue A, Kobayashi K, et al.; for North-East Japan Study Group. Gefitinib or chemotherapy for non-small-cell lung cancer with mutated EGFR. $N$ Engl $J$ Med. 2010;362 (25):2380-2388. doi:10.1056/NEJMoa0909530

33. Sculier JP, Chansky K, Crowley JJ, Van Meerbeeck J, Goldstraw P; for International Staging Committee and Participating Institutions. The impact of additional prognostic factors on survival and their relationship with the anatomical extent of disease expressed by the 6th edition of the TNM classification of malignant tumors and the proposals for the 7th edition. $J$ Thorac Oncol. 2008;3(5):457-466. doi:10.1097/JTO.0b013e31816de2b8

34. Granger CL, McDonald CF, Irving L, et al. Low physical activity levels and functional decline in individuals with lung cancer. Lung Cancer. 2014;83(2):292-299. doi:10.1016/j.lungcan.2013.11.014

35. Fujisawa D, Temel JS, Greer JA, et al. Actigraphy as an assessment of performance status in patients with advanced lung cancer. Palliat Support Care. 2019;17(5):574-578. doi:10.1017/ S1478951518001074

36. Ohri N, Halmos B, Bodner WR, et al. Daily step counts: a new prognostic factor in locally advanced non-small cell lung cancer? Int J Radiat Oncol Biol Phys. 2019;105(4):745-751. doi:10.1016/j. ijrobp.2019.07.055

37. Norman K, Otten L. Financial impact of sarcopenia or low muscle mass - A short review. Clin Nutr. 2019;38(4):1489-1495. doi:10.1016/j.clnu.2018.09.026 
38. Vermillion SA, Hsu FC, Dorrell RD, Shen P, Clark CJ. Modified frailty index predicts postoperative outcomes in older gastrointestinal cancer patients. J Surg Oncol. 2017;115(8):997-1003. doi:10.1002/ jso. 24617

39. Murphy CC, Incalcaterra JR, Albright HW, Correa AM, Swisher SG, Hofstetter WL. Pretreatment patient comorbidity and tobacco use increase cost and risk of postoperative complications after esophagectomy at a high-volume cancer center. J Oncol Pract. 2013;9 (5):233-239. doi:10.1200/JOP.2013.001047

40. Ohri N, Kabarriti R, Bodner WR, et al. Continuous activity monitoring during concurrent chemoradiotherapy. Int J Radiat Oncol Biol Phys. 2017;97(5):1061-1065. doi:10.1016/j.ijrobp.2016.12.030

41. Douma JAJ, de Beaufort MB, Kampshoff CS, et al. Physical activity in patients with cancer: self-report versus accelerometer assessments. Support Care Cancer. 2020;28(8):3701-3709. doi:10.1007/s00520019-05203-3

42. Granger CL, Parry SM, Denehy L. The self-reported Physical Activity Scale for the Elderly (PASE) is a valid and clinically applicable measure in lung cancer. Support Care Cancer. 2015;23 (11):3211-3218. doi:10.1007/s00520-015-2707-8

43. Giné-Garriga M, Roqué-Fíguls M, Coll-Planas L, Sitjà-Rabert M, Salvà A. Physical exercise interventions for improving performance-based measures of physical function in community-dwelling, frail older adults: a systematic review and meta-analysis. Arch Phys Med Rehabil. 2014;95(4):753-769.e3. doi:10.1016/j.apmr.2013.11.007

44. Avancini A, Tregnago D, Rigatti L, et al. Factors influencing physical activity in cancer patients during oncological treatments: a Qualitative Study. Integr Cancer Ther. 2020;19:1534735420971365. doi:10.1177/ 1534735420971365
45. Avancini A, Sartori G, Gkountakos A, et al. Physical activity and exercise in lung cancer care: will promises be fulfilled? Oncologist. 2019; theoncologist.2019-0463.

46. Heywood R, McCarthy AL, Skinner TL. Efficacy of exercise interventions in patients with advanced cancer: a systematic review. Arch Phys Med Rehabil. 2018;99(12):2595-2620. doi:10.1016/j. apmr.2018.04.008

47. Dittus KL, Gramling RE, Ades PA. Exercise interventions for individuals with advanced cancer: a systematic review. Prev Med. 2017;104:124-132. doi:10.1016/j.ypmed.2017.07.015

48. Grande GD, Oliveira CB, Morelhão PK, et al. Interventions promoting physical activity among older adults: a systematic review and meta-analysis. Gerontologist. 2020;60(8):583-599. doi:10.1093/geront/gnz167

49. Schaffer K, Panneerselvam N, Loh KP, et al. Systematic review of randomized controlled trials of exercise interventions using digital activity trackers in patients with cancer. $J$ Natl Compr Canc Netw. 2019;17(1):57-63. doi:10.6004/jnccn.2018.7082

50. Miura S, Naito T, Mitsunaga S, et al. A randomized Phase II study of nutritional and exercise treatment for elderly patients with advanced non-small cell lung or pancreatic cancer: the NEXTAC-TWO Study protocol. BMC Cancer. 2019;19(1):528. doi:10.1186/s12885-019$5762-6$
Journal of Multidisciplinary Healthcare

\section{Publish your work in this journal}

The Journal of Multidisciplinary Healthcare is an international, peerreviewed open-access journal that aims to represent and publish research in healthcare areas delivered by practitioners of different disciplines. This includes studies and reviews conducted by multidisciplinary teams as well as research which evaluates the results or conduct of such teams or healthcare processes in general. The journal

\section{Dovepress}

covers a very wide range of areas and welcomes submissions from practitioners at all levels, from all over the world. The manuscript management system is completely online and includes a very quick and fair peer-review system. Visit http://www.dovepress.com/testimonials. php to read real quotes from published authors. 